\title{
The geometric rate of convergence of random iteration in the Hutchinson distance
}

\author{
RAFAE KAPICA
}

Dedicated to Professor Karol Baron on his 70th birthday.

\begin{abstract}
Using the Banach fixed-point theorem we provide a simple criterion of the geometric rate of convergence and of asymptotic stability of Markov operators in the Hutchinson distance. The obtained results are applied to sequences of iterates of random-valued functions.
\end{abstract}

Mathematics Subject Classification. Primary 60J05, Secondary 37A99.

Keywords. Markov operators, Random-valued functions, Random iteration, Asymptotic stability, Rate of convergence.

\section{Introduction}

In 1995 Lasota [10] established some sufficient conditions for the asymptotic stability of Markov Feller operators acting on a metric space and obtained two nice criteria for Markov operators generated by iterated function systems. The first criterion was based on the so called double contraction principle discovered by Lasota, which is a generalization of the classical Banach fixed-point theorem. Following an idea from [10] Baron has used the Hutchinson distance of distributions to get the geometric rate of convergence of sequences of distributions of iterates of random valued vector functions in the Fortet-Mourier metric (see [1]). Recently this result has been strengthened in [2] by the fact that the distribution of the limit has the first moment finite. The aim of the present paper, motivated by $[1,10]$, is to show how fast a sequence of iterates of Markov operators tends in the Hutchinson distance to a unique invariant and attractive measure. Moreover, as an application of our main result we will examine a speed of convergence of sequences of iterates of random-valued functions having values in metric spaces and adopting a definition of iterates 
from [4]. To get our criterion of the geometric rate and asymptotic stability of Markov operators in the Hutchinson distance we will use the Banach fixed-point theorem. It turns out that by the use of that classical contraction mapping principle we give stronger results under the same assumptions than in the listed papers. Namely, Theorem 4.1 and Corollary 5.6 given below generalize [10, Theorem 3.2] and [1, Theorem 3.1], respectively.

\section{Notions and basic facts}

Assume that $(X, \varrho)$ is a Polish space, i.e. a separable and complete metric space. Let $\mathcal{B}(X)$ stand for the $\sigma$-algebra of all Borel subsets of $X$. By $\mathcal{M}_{1}(X)$ we denote the space of all probability measures on $\mathcal{B}(X)$. Let $B(X)$ denote the space of all bounded Borel-measurable functions equipped with the supremum norm $\|\cdot\|_{\infty}$ and $C(X)$ be the subspace of bounded continuous functions. For brevity we will write $\int \varphi d \mu$ instead of $\int_{X} \varphi d \mu$, where $\varphi \in B(X)$ and $\mu \in \mathcal{M}_{1}(X)$. Recall that a sequence of measures $\left(\mu_{n}\right)$ converges weakly to $\mu$, if $\int \varphi d \mu_{n} \underset{n \rightarrow \infty}{\longrightarrow} \int \varphi d \mu$ for every $\varphi \in C(X)$. It is well known (see [6, Theorem 11.3.3]) that this convergence is metrizable by the Fortet-Mourier (known also as Lévy-Prokhorov) metric [7]

$$
d_{F M}(\mu, \nu)=\sup \left\{\left|\int \varphi d \mu-\int \varphi d \nu\right|: \varphi \in \operatorname{Lip}_{1}(X),\|\varphi\|_{\infty} \leq 1\right\},
$$

where

$$
\operatorname{Lip}_{1}(X)=\{\varphi: X \rightarrow \mathbb{R}:|\varphi(x)-\varphi(y)| \leq \varrho(x, y), x, y \in X\} .
$$

Putting $\operatorname{Lip}_{1}^{b}(X)=\operatorname{Lip}_{1}(X) \cap B(X)$ define now

$$
d_{H}(\mu, \nu)=\sup \left\{\left|\int \varphi d \mu-\int \varphi d \nu\right|: \varphi \in \operatorname{Lip}_{1}^{b}(X)\right\} .
$$

Clearly, $d_{H}$ is a distance function, called the Hutchinson metric [8] (also called the 1-Wasserstein distance or Kantorovich-Rubinstein distance), however for some arguments it may be infinite. Moreover $d_{F M}(\mu, \nu) \leq d_{H}(\mu, \nu)$ and in the case when the space $X$ is bounded we have $d_{H}(\mu, \nu) \leq \operatorname{diam}(X) d_{F M}(\mu, \nu)$ for any $\mu, \nu \in \mathcal{M}_{1}(X)$, i.e. metrics $d_{F M}, d_{H}$ are equivalent. For more details concerning metrics on measures we refer the reader to $[6,11]$.

Throughout this paper we shall consider a regular Markov operator $P$ : $\mathcal{M}_{1}(X) \rightarrow \mathcal{M}_{1}(X)$, i.e. $P$ is a linear operator (here linearity is restricted to nonnegative and summing up to one coefficients only) and there exists an (adjoint or dual) operator $P^{*}: B(X) \rightarrow B(X)$ such that $\int \varphi d P \mu=\int P^{*} \varphi d \mu$ for any $\varphi \in B(X)$ and $\mu \in \mathcal{M}_{1}(X)$. Moreover, if $P^{*}: B(X) \rightarrow B(X)$ is a linear operator, $P^{*} \mathbf{1}_{X}=\mathbf{1}_{X}$ and $P^{*} \varphi \geq 0$ if $\varphi \geq 0$, then the operator $P$ given by $P \mu(A)=\int P^{*} \mathbf{1}_{A}(x) \mu(d x), A \in \mathcal{B}(X)$, is the Markov operator (with adjoint $\left.P^{*}\right)$. 
Assume that $(\Omega, \mathcal{A}, \mathbb{P})$ is a probability space. A function $f: X \times \Omega \rightarrow X$ is said to be a random-valued function (shortly rv-function) if it is measurable with respect to the product $\sigma$-algebra $\mathcal{B}(X) \otimes \mathcal{A}$. Having an rv-function $f$ we will examine a regular Markov operator $P: \mathcal{M}_{1}(X) \rightarrow \mathcal{M}_{1}(X)$ defined by

$$
P \mu(A)=\int_{X} \int_{\Omega} \mathbf{1}_{A}(f(x, \omega)) \mathbb{P}(d \omega) \mu(d x), \quad \mu \in \mathcal{M}_{1}(X), A \in \mathcal{B}(X) .
$$

One can show that $P$ is a transition operator for a sequence of iterates of rv-functions in the sense of Baron and Kuczma [4] (cf. [5]). More precisely, $P \pi_{n}(x, \cdot)=\pi_{n+1}(x, \cdot)$, where $\pi_{n}(x, B)=\mathbb{P}^{\infty}\left(f^{n}(x, \cdot) \in B\right), B \in \mathcal{B}(X)$, denotes the distribution of the $n$th iterate of $f$ defined inductively as follows

$$
f^{1}(x, \omega)=f\left(x, \omega_{1}\right), \quad f^{n+1}(x, \omega)=f\left(f^{n}(x, \omega), \omega_{n+1}\right),
$$

for $x$ from $X$ and $\omega=\left(\omega_{1}, \omega_{2}, \ldots\right)$ from $\Omega^{\infty}$ being $\Omega^{\mathbb{N}}$. Note that $f^{n}: X \times$ $\Omega^{\infty} \rightarrow X$ is an rv-function on the product probability space $\left(\Omega^{\infty}, \mathcal{A}^{\infty}, \mathbb{P}^{\infty}\right)$. More exactly, the $n$th iterate $f^{n}$ is $\mathcal{B}(X) \otimes \mathcal{A}_{n}$-measurable, where $\mathcal{A}_{n}$ denotes the $\sigma$-algebra of all the sets of the form $\left\{\left(\omega_{1}, \omega_{2}, \ldots\right) \in \Omega^{\infty}:\left(\omega_{1}, \omega_{2}, \ldots, \omega_{n}\right) \in\right.$ $A\}$ with $A$ from the product $\sigma$-algebra $\mathcal{A}^{n}$.

\section{Properties of some family of measures}

Let $(X, \varrho)$ be a Polish space. Assume that $\mu_{0} \in \mathcal{M}_{1}(X)$ is fixed and let us consider a family $\mathcal{M}\left(\mu_{0}\right)$ given by

$$
\mathcal{M}\left(\mu_{0}\right)=\left\{\mu \in \mathcal{M}_{1}(X): d_{H}\left(\mu, \mu_{0}\right)<\infty\right\} .
$$

Clearly, if $\mu \in \mathcal{M}\left(\mu_{0}\right)$, then $d_{H}(\mu, \nu)<\infty$ if and only if $\nu \in \mathcal{M}\left(\mu_{0}\right)$. In other words the set $\mathcal{M}\left(\mu_{0}\right)$ does not depend on the choice of the measure $\mu_{0}$ in the following sense:

$$
\text { if } d_{H}\left(\mu_{0}, \nu_{0}\right)<\infty \text {, then } \mathcal{M}\left(\mu_{0}\right)=\mathcal{M}\left(\nu_{0}\right) \text {. }
$$

Since $d_{H}\left(\delta_{x}, \delta_{y}\right)=\rho(x, y)$, it follows that $\mathcal{M}\left(\delta_{x}\right)=\mathcal{M}\left(\delta_{y}\right)$ for any $x, y \in X$. (By $\delta_{x}$ we mean the Dirac measure concentrated at $x \in X$.) We will show that $\mathcal{M}\left(\delta_{x_{0}}\right)$ is equal to the family $\mathcal{M}_{1}^{1}(X)$ defined by

$$
\mathcal{M}_{1}^{1}(X)=\left\{\mu \in \mathcal{M}_{1}(X): \int \varrho\left(x, x_{0}\right) \mu(d x)<\infty\right\}
$$

for some $x_{0} \in X$; see Lemma 3.1 given below. Note that the set $\mathcal{M}_{1}^{1}(X)$ is independent of $x_{0}$ and it consists of all Borel measures on $X$ with the first moment finite.

Lemma 3.1. Assume that $\mu \in \mathcal{M}_{1}^{1}(X)$ and $x_{0} \in X$. 
(i) If $\nu \in \mathcal{M}_{1}^{1}(X)$, then $d_{H}(\mu, \nu)=D_{H}(\mu, \nu)<\infty$, where

$$
D_{H}(\mu, \nu)=\sup \left\{\int \varphi d \mu-\int \varphi d \nu: \varphi \in \operatorname{Lip}_{1}(X)\right\} .
$$

Moreover, $d_{H}\left(\mu, \delta_{x_{0}}\right)=D_{H}\left(\mu, \delta_{x_{0}}\right)=\int \varrho\left(x, x_{0}\right) \mu(d x)$.

(ii) If $\nu \in \mathcal{M}_{1}(X)$ and $d_{H}\left(\nu, \delta_{x_{0}}\right)<\infty$, then $\nu \in \mathcal{M}_{1}^{1}(X)$.

Proof. (i) Fix $\varphi \in \operatorname{Lip}_{1}(X)$ and observe that

$$
\int|\varphi(x)| \mu(d x) \leq \int \varrho\left(x, x_{0}\right) \mu(d x)+\left|\varphi\left(x_{0}\right)\right|<\infty .
$$

Clearly $D_{H}(\mu, \nu)=\sup \left\{\left|\int \psi d \mu-\int \psi d \nu\right|: \psi \in \operatorname{Lip}_{1}(X)\right\}$, so $d_{H}(\mu, \nu) \leq$ $D_{H}(\mu, \nu)$. To prove $d_{H}(\mu, \nu) \geq D_{H}(\mu, \nu)$, it is enough to show that

$$
d_{H}(\mu, \nu) \geq\left|\int \varphi d \mu-\int \varphi d \nu\right| \text {. }
$$

To do this let us consider $\varphi_{n} \in \operatorname{Lip}_{1}^{b}(X), n \in \mathbb{N}$, given by

$$
\varphi_{n}(x)=\left\{\begin{array}{ll}
n, & \varphi(x)>n \\
\varphi(x), & |\varphi(x)| \leq n \\
-n, & \varphi(x)<-n
\end{array} .\right.
$$

Obviously, the sequence $\left(\varphi_{n}\right)$ converges pointwise to $\varphi$, and by the Lebesgue dominated theorem we have

$$
\left|\int \varphi_{n} d \mu-\int \varphi_{n} d \nu\right| \underset{n \rightarrow \infty}{\longrightarrow}\left|\int \varphi d \mu-\int \varphi d \nu\right| .
$$

Thus we get

$$
d_{H}(\mu, \nu) \geq \sup _{n \in \mathbb{N}}\left|\int \varphi_{n} d \mu-\int \varphi_{n} d \nu\right| \geq\left|\int \varphi d \mu-\int \varphi d \nu\right|,
$$

as expected.

Now let us define a Lyapunov function $V: X \rightarrow[0, \infty)$ by

$$
V(x)=\varrho\left(x, x_{0}\right) .
$$

Then $V \in \operatorname{Lip}_{1}(X)$ and for every $\varphi \in \operatorname{Lip}_{1}(X)$ we have

$$
\begin{aligned}
\int \varphi d \mu-\int \varphi d \delta_{x_{0}} & =\int\left(\varphi(x)-\varphi\left(x_{0}\right)\right) \mu(d x) \\
& \leq \int V d \mu=\int V d \mu-\int V d \delta_{x_{0}} \leq D_{H}\left(\mu, \delta_{x_{0}}\right) .
\end{aligned}
$$

Hence $D_{H}\left(\mu, \delta_{x_{0}}\right)=\int V d \mu$.

(ii) Define $V_{n} \in \operatorname{Lip}_{1}^{b}(X)$ by

$$
V_{n}(x)=\left\{\begin{array}{ll}
n, & V(x)>n \\
V(x), & V(x) \leq n
\end{array},\right.
$$


where $V$ is given by (3.2). Hence the sequence $\left(V_{n}\right)$ is increasing and convergent to $V$, and

$$
\int V d \nu=\lim _{n \rightarrow \infty} \int V_{n} d \nu=\lim _{n \rightarrow \infty}\left(\int V_{n} d \nu-\int V_{n} d \delta_{x_{0}}\right) \leq d_{H}\left(\nu, \delta_{x_{0}}\right) .
$$

Remark 3.2. Assume that $\mu_{0} \in \mathcal{M}_{1}(X)$. Then $\mathcal{M}\left(\mu_{0}\right)=\mathcal{M}_{1}^{1}(X)$ if and only if $\mu_{0} \in \mathcal{M}_{1}^{1}(X)$. In particular $\mathcal{M}_{1}^{1}(X)=\mathcal{M}\left(\delta_{x_{0}}\right)$.

The above remark follows immediately from Lemma 3.1 and (3.1), and it suggests that the set $\mathcal{M}\left(\mu_{0}\right)$ with metric $d_{H}$ forms a complete space as $\left(\mathcal{M}_{1}^{1}(X), D_{H}\right)$ does (see [11, Theorem 6.18]). In fact, we have the following theorem concerning the topological properties of $\mathcal{M}\left(\mu_{0}\right)$.

Theorem 3.3. Assume that $\mu_{0} \in \mathcal{M}_{1}(X)$. Then $\left(\mathcal{M}\left(\mu_{0}\right), d_{H}\right)$ is a complete metric space and the set $\mathcal{M}\left(\mu_{0}\right)$ is a dense subset of the space $\mathcal{M}_{1}(X)$ with the Fortet-Mourier metric.

Proof. The fact that $d_{H}$ is a (finite) metric on $\mathcal{M}\left(\mu_{0}\right)$ was already explained, so let us turn to the proof of completeness. To do this fix a Cauchy sequence $\left(\mu_{n}\right)$ in $\left(\mathcal{M}\left(\mu_{0}\right), d_{H}\right)$ and observe that $d_{F M}\left(\mu_{n}, \mu_{m}\right) \leq d_{H}\left(\mu_{n}, \mu_{m}\right)$, which shows that $\left(\mu_{n}\right)$ is a Cauchy sequence in a space $\mathcal{M}_{1}(X)$ with the Fortet-Mourier metric $d_{F M}$. Since this metric is complete it follows that $\left(\mu_{n}\right)$ converges in $d_{F M}$, or equivalently $\left(\mu_{n}\right)$ is weakly convergent, say to $\mu \in \mathcal{M}_{1}(X)$. Fix $\varepsilon>0$ and assume that $n_{0} \in \mathbb{N}$ is such that

$$
d_{H}\left(\mu_{n}, \mu_{m}\right)<\varepsilon \text { for } n, m \geq n_{0} .
$$

Then for the same $n, m$, and for an arbitrarily fixed $\varphi \in \operatorname{Lip}_{1}^{b}(X)$ we have

$$
\left|\int \varphi d \mu-\int \varphi d \mu_{n}\right| \leq\left|\int \varphi d \mu-\int \varphi d \mu_{m}\right|+d_{H}\left(\mu_{m}, \mu_{n}\right) .
$$

Consequently for any fixed $n \geq n_{0}$

$$
\left|\int \varphi d \mu-\int \varphi d \mu_{n}\right| \leq\left|\int \varphi d \mu-\int \varphi d \mu_{m}\right|+\varepsilon \quad \text { for } m \geq n_{0},
$$

and passing with $m$ to infinity we see that

$$
\left|\int \varphi d \mu-\int \varphi d \mu_{n}\right| \leq \varepsilon \quad \text { for any } n \geq n_{0} .
$$

Therefore

$$
d_{H}\left(\mu, \mu_{n}\right) \leq \varepsilon \quad \text { for any } n \geq n_{0},
$$

which means that $\mu_{n} \underset{n \rightarrow \infty}{\longrightarrow} \mu$ in metric $d_{H}$ and to finish the first part of our proof it is enough to observe that $\mu \in \mathcal{M}\left(\mu_{0}\right)$. However, since $\mu_{n} \in \mathcal{M}\left(\mu_{0}\right)$, we see that $d_{H}\left(\mu, \mu_{0}\right)<\infty$. 
A proof of the density of $\mathcal{M}\left(\mu_{0}\right)$ is, in fact, a part of a proof of $[10$, Theorem 3.2 . For the convenience of the reader we will show it based on ideas from that paper. Fix $\mu \in \mathcal{M}_{1}(X), x_{0} \in X$, and let $r$ be any positive real such that $\mu\left(B_{r}\right)>0$, where $B_{r}$ is an open ball centered at $x_{0}$ with radius $r$. Define a measure $\mu_{r} \in \mathcal{M}_{1}(X)$ by

$$
\mu_{r}(A)=c_{r} \mu\left(A \cap B_{r}\right)+\mu_{0}\left(A \cap B_{r}^{\prime}\right),
$$

where $c_{r}=\mu_{0}\left(B_{r}\right) / \mu\left(B_{r}\right)$ and $B_{r}^{\prime}=X \backslash B_{r}$. We will show that $\mu_{r} \in \mathcal{M}\left(\mu_{0}\right)$. To this end fix $\varphi \in \operatorname{Lip}_{1}^{b}(X)$ and note that

$$
\begin{aligned}
\left|\int \varphi d \mu_{r}-\int \varphi d \mu_{0}\right|= & \left|\int_{B_{r}} c_{r} \varphi d \mu-\int_{B_{r}} \varphi d \mu_{0}\right| \\
= & \mid \int_{B_{r}} c_{r}\left(\varphi(x)-\varphi\left(x_{0}\right)\right) \mu(d x)+c_{r} \varphi\left(x_{0}\right) \mu\left(B_{r}\right) \\
& -\left(\int_{B_{r}}\left(\varphi(x)-\varphi\left(x_{0}\right)\right) \mu_{0}(d x)+\varphi\left(x_{0}\right) \mu_{0}\left(B_{r}\right)\right) \mid \\
= & \mid \int_{B_{r}} c_{r}\left(\varphi(x)-\varphi\left(x_{0}\right)\right) \mu(d x)-\int_{B_{r}}\left(\varphi(x)-\varphi\left(x_{0}\right)\right) \mu_{0}(d x) \\
& +\frac{\mu_{0}\left(B_{r}\right)}{\mu\left(B_{r}\right)} \varphi\left(x_{0}\right) \mu\left(B_{r}\right)-\varphi\left(x_{0}\right) \mu_{0}\left(B_{r}\right) \mid \\
\leq & c_{r} \int_{B_{r}}\left|\varphi(x)-\varphi\left(x_{0}\right)\right| \mu(d x)+\int_{B_{r}}\left|\varphi(x)-\varphi\left(x_{0}\right)\right| \mu_{0}(d x) \\
< & c_{r} r \mu\left(B_{r}\right)+r \mu_{0}\left(B_{r}\right)=2 r \mu_{0}\left(B_{r}\right) .
\end{aligned}
$$

Thus $d_{H}\left(\mu_{r}, \mu_{0}\right) \leq 2 r \mu_{0}\left(B_{r}\right)<\infty$, i.e. $\mu_{r} \in \mathcal{M}\left(\mu_{0}\right)$. (Clearly $\mu_{r^{\prime}} \in \mathcal{M}\left(\mu_{0}\right)$ for $r^{\prime}>r$.)

Now for any $\varphi \in \operatorname{Lip}_{1}(X),\|\varphi\|_{\infty} \leq 1$ we have

$$
\begin{aligned}
\left|\int \varphi d \mu_{r}-\int \varphi d \mu\right| & \leq \int_{B_{r}}|\varphi|\left|c_{r}-1\right| d \mu+\int_{B_{r}^{\prime}}|\varphi| d \mu_{0}+\int_{B_{r}^{\prime}}|\varphi| d \mu \\
& \leq\left|c_{r}-1\right| \mu\left(B_{r}\right)+\mu_{0}\left(B_{r}^{\prime}\right)+\mu\left(B_{r}^{\prime}\right) \underset{r \rightarrow \infty}{\longrightarrow} 0,
\end{aligned}
$$

hence $d_{F M}\left(\mu_{r}, \mu\right) \underset{r \rightarrow \infty}{\longrightarrow} 0$, which finishes the proof.

\section{Asymptotic stability and the rate of convergence of Markov operators}

Let $(X, \varrho)$ be a Polish space. We say that a Markov operator $P: \mathcal{M}_{1}(X) \rightarrow$ $\mathcal{M}_{1}(X)$ is asymptotically stable, if it has an invariant and attractive measure. Strictly speaking, $\mu^{*} \in \mathcal{M}_{1}(X)$ is invariant for $P$ if $P \mu^{*}=\mu^{*}$ or alternatively $\int P^{*} \varphi d \mu^{*}=\int \varphi d \mu^{*}$ for every $\varphi \in B(X)$; respectively, $\mu^{*}$ is attractive for $P$ if $P^{n} \mu \underset{n \rightarrow \infty}{\stackrel{w}{\longrightarrow}} \mu^{*}$ for every $\mu \in \mathcal{M}_{1}(X)$, where $P^{1}=P, P^{n+1}=P^{n} \circ P$ 
and the limit is in a weak sense. Clearly, if $P$ is asymptotically stable then $P$ has exactly one invariant measure. The main result of this section concerns a regular Markov operator and it reads as follows.

Theorem 4.1. Let $P: \mathcal{M}_{1}(X) \rightarrow \mathcal{M}_{1}(X)$ be a regular Markov operator with adjoint operator $P^{*}: B(X) \rightarrow B(X)$. Assume that there exists $\lambda \in(0,1)$ for which

$$
\left|P^{*} \varphi(x)-P^{*} \varphi(y)\right| \leq \lambda \varrho(x, y) \quad \text { for } x, y \in X \text { and } \varphi \in \operatorname{Lip}_{1}^{b}(X) .
$$

Assume moreover that

$$
d_{H}\left(\mu_{0}, P \mu_{0}\right)<\infty
$$

for some $\mu_{0} \in \mathcal{M}_{1}(X)$. Then the operator $P$ is asymptotically stable and its invariant measure $\mu^{*}$ belongs to $\mathcal{M}\left(\mu_{0}\right)$. Moreover we have a geometric rate of convergence, i.e

$$
d_{H}\left(P^{n} \mu, \mu^{*}\right) \leq \frac{\lambda^{n}}{1-\lambda} d_{H}(\mu, P \mu)
$$

for any $n \in \mathbb{N}$ and $\mu \in \mathcal{M}\left(\mu_{0}\right)$.

The proof of Theorem 4.1 will be preceded by a few results concerning the assumptions of the theorem.

Remark 4.2. There is no need for the assumption in Theorem 4.1 as well as in the following facts of the paper that the operator $P$ has the Feller property, i.e. $P^{*}(C(X)) \subset C(X)$, which implies that such an operator is continuous in the topology of weak convergence. The property considered here was assumed in the main results of [10].

Lemma 4.3. Assume that an operator $P$ with adjoint $P^{*}$ satisfies condition (4.1) with some $\lambda \in(0,1)$. Then the following assertions hold true:

$$
d_{H}(P \mu, P \nu) \leq \lambda d_{H}(\mu, \nu) \quad \text { for } \mu, \nu \in \mathcal{M}_{1}(X),
$$

(ii) for $\mu_{0} \in \mathcal{M}_{1}(X)$ we have

$$
P\left(\mathcal{M}\left(\mu_{0}\right)\right) \subset \mathcal{M}\left(\mu_{0}\right)
$$

if and only if $\mu_{0}$ satisfies condition (4.2).

Proof. Fix $\mu, \nu \in \mathcal{M}_{1}(X)$ and $\varphi \in \operatorname{Lip}_{1}^{b}(X)$. Then $P^{*} \varphi \in B(X)$ and $\frac{1}{\lambda} P^{*} \varphi \in$ $\operatorname{Lip}_{1}(X)$ by (4.1), and hence

$$
\left|\int \varphi d P \mu-\int \varphi d P \nu\right|=\lambda\left|\int \frac{1}{\lambda} P^{*} \varphi d \mu-\int \frac{1}{\lambda} P^{*} \varphi d \nu\right| \leq \lambda d_{H}(\mu, \nu),
$$

which shows (4.4).

Assertion (ii) follows from (4.4) and (4.2) easily. 
Proposition 4.4. Assume that an operator $P$ with adjoint $P^{*}$ satisfies condition (4.1) with some $\lambda \in(0,1)$ and let $\mu_{0} \in \mathcal{M}_{1}(X)$. Then the following assertions are pairwise equivalent:

(i) $P\left(\mathcal{M}\left(\mu_{0}\right)\right) \subset \mathcal{M}\left(\mu_{0}\right)$,

(ii) $\quad d_{H}(P \mu, \mu)<\infty$ for every $\mu \in \mathcal{M}\left(\mu_{0}\right)$,

(iii) $\quad d_{H}(P \mu, \mu)<\infty$ for some $\mu \in \mathcal{M}\left(\mu_{0}\right)$.

The above proposition is an immediate consequence of Lemma 4.3 and jointly with Remark 3.2 implies the following fact.

Corollary 4.5. Assume that an operator $P$ with adjoint $P^{*}$ satisfies condition (4.1) with some $\lambda \in(0,1)$. Then the following assertions are pairwise equivalent:

(i) $\quad P\left(\mathcal{M}_{1}^{1}(X)\right) \subset \mathcal{M}_{1}^{1}(X)$,

(ii) $\quad d_{H}(P \mu, \mu)<\infty$ for every $\mu \in \mathcal{M}_{1}^{1}(X)$,

(iii) $\quad d_{H}(P \mu, \mu)<\infty$ for some $\mu \in \mathcal{M}_{1}^{1}(X)$,

(iv) $\quad d_{H}\left(P \delta_{x}, \delta_{x}\right)<\infty$ for every $x \in X$,

(v) $\quad d_{H}\left(P \delta_{x}, \delta_{x}\right)<\infty$ for some $x \in X$.

Proof of Theorem 4.1. From Lemma 4.3 and Theorem 3.3 it follows that $P$ restricted to $\mathcal{M}\left(\mu_{0}\right)$ is a $\lambda$-contraction acting on a complete metric space $\mathcal{M}\left(\mu_{0}\right)$ into itself. By the Banach fixed-point theorem there exists a unique invariant measure $\mu^{*} \in \mathcal{M}\left(\mu_{0}\right)$ for $P$ and condition (4.3) holds.

It remains to prove the asymptotic stability of $P$. To this end observe first that $P$ is a non-expanding operator with respect to the Fortet-Mourier metric. Indeed, if $\varphi \in \operatorname{Lip}_{1}(X)$ and $\|\varphi\|_{\infty} \leq 1$, then

$$
\left|P^{*} \varphi(x)\right|=\left|\int P^{*} \varphi d \delta_{x}\right| \leq \int|\varphi| d P \delta_{x} \leq 1, \quad\left|P^{*} \varphi(x)-P^{*} \varphi(y)\right| \leq \varrho(x, y)
$$

for any $x, y \in X$, which shows that $P^{*} \varphi \in \operatorname{Lip}_{1}(X),\left\|P^{*} \varphi\right\|_{\infty} \leq 1$ and gives

$$
\left|\int \varphi d P \mu-\int \varphi d P \nu\right|=\left|\int P^{*} \varphi d \mu-\int P^{*} \varphi d \nu\right| \leq d_{F M}(\mu, \nu)
$$

for $\mu, \nu \in \mathcal{M}_{1}(X)$, i.e.

$$
d_{F M}(P \mu, P \nu) \leq d_{F M}(\mu, \nu) .
$$

Fix $\mu \in \mathcal{M}_{1}(X)$ and $\varepsilon>0$. According to Theorem 3.3 there is $\nu \in \mathcal{M}\left(\mu_{0}\right)$ such that $d_{F M}(\mu, \nu)<\varepsilon$. By (4.3) we have $d_{H}\left(P^{n} \nu, \mu^{*}\right)<\varepsilon$ for every large enough $n \in \mathbb{N}$. Then for such $n$ we obtain

$$
\begin{aligned}
d_{F M}\left(P^{n} \mu, \mu^{*}\right) & \leq d_{F M}\left(P^{n} \mu, P^{n} \nu\right)+d_{F M}\left(P^{n} \nu, \mu^{*}\right) \\
& \leq d_{F M}(\mu, \nu)+d_{H}\left(P^{n} \nu, \mu^{*}\right)<2 \varepsilon .
\end{aligned}
$$




\section{Application to the iterates of random-valued functions}

Let $(X, \varrho)$ be a Polish space. Assume that $(\Omega, \mathcal{A}, \mathbb{P})$ is a probability space and $f: X \times \Omega \rightarrow X$ is an $r v$-function. We will examine a transition operator (2.1) for distributions $\pi_{n}(x, \cdot)$ of iterates $(2.2)$. Putting

$$
\pi(x, B)=\pi_{1}(x, B)=\mathbb{P}(f(x, \cdot) \in B) \quad \text { for any } x \in X, B \in \mathcal{B}(X),
$$

we observe that $\pi(x, \cdot)$ is a probability measure on $\mathcal{B}(X)$ and $\pi(\cdot, B)$ is a Borelmeasurable function for any fixed $x \in X$ and $B \in \mathcal{B}(X)$. This allows us to show that the operator $P^{*}$ given by

$$
P^{*} \varphi(x)=\int_{\Omega} \varphi(f(x, \omega)) \mathbb{P}(d \omega), \quad \varphi \in B(X), x \in X,
$$

is adjoint to (2.1), and in addition,

$$
\pi_{n}(x, B)=P^{* n} \mathbf{1}_{B}(x)=P^{n} \delta_{x}(B)
$$

for any $x \in X$ and $B \in \mathcal{B}(X)$.

Remark 5.1. We say that an rv-function $f: X \times \Omega \rightarrow X$ is $\mathbb{P}$-continuous (see [3]), if for every sequence $\left(x_{j}\right)$ of points from $X$ tending to $x \in X$, the sequence $\left(f\left(x_{j}, \cdot\right)\right)$ converges in probability $\mathbb{P}$ to $f(x, \cdot)$. One can show that $P$ given by (2.1) with $\mathbb{P}$-continuous $f$ is a regular Markov Feller operator and it is asymptotically stable if and only if for every $x \in X$ the sequence $\left(f^{n}(x, \cdot)\right)$ converges in distribution or in law (which means that $\left(\pi_{n}(x, \cdot)\right)$ converges weakly) and the limit $\mu^{*}$ does not depend on $x$; see [9, Proposition 2.1 and Theorem 2.3]. Moreover, the measure $\mu^{*}$ is an invariant measure for the operator $P$.

Following [1] we shall consider two conditions:

$$
\int_{\Omega} \rho(f(x, \omega), f(y, \omega)) \mathbb{P}(d \omega) \leq \lambda \rho(x, y) \quad \text { for } x, y \in X,
$$

and

$$
\int_{\Omega} \rho\left(f\left(x_{0}, \omega\right), x_{0}\right) \mathbb{P}(d \omega)<\infty
$$

with $\lambda \in(0, \infty)$ and for some (thus all) $x_{0} \in X$.

Remark 5.2. Assume that (5.2) and (5.3) hold for some $\lambda \in(0, \infty)$ and $x_{0} \in X$, and let $\mu \in \mathcal{M}_{1}^{1}(X)$. Then

$$
\begin{aligned}
\int_{X} \int_{\Omega} \rho(f(x, \omega), x) \mathbb{P}(d \omega) \mu(d x) \leq & (\lambda+1) \int_{X} \rho\left(x, x_{0}\right) \mu(d x) \\
& +\int_{\Omega} \rho\left(f\left(x_{0}, \omega\right), x_{0}\right) \mathbb{P}(d \omega)
\end{aligned}
$$

for every $x \in X$. Similarly,

$$
\int_{X} \rho\left(x, x_{0}\right) \mu(d x) \leq \frac{1}{1-\lambda}\left(\int_{X} \int_{\Omega} \rho(f(x, \omega), x) \mathbb{P}(d \omega) \mu(d x)\right.
$$




$$
\left.+\int_{\Omega} \rho\left(f\left(x_{0}, \omega\right), x_{0}\right) \mathbb{P}(d \omega)\right)
$$

for every $x \in X$, provided $\lambda \in(0,1)$.

Lemma 5.3. Assume that (5.2) and (5.3) hold for some $\lambda \in(0,1)$ and $x_{0} \in X$, and let $\mu \in \mathcal{M}_{1}^{1}(X)$. Then the operator $P$ given by (2.1) satisfies

$$
d_{H}(P \mu, \mu) \leq \int_{X} \int_{\Omega} \rho(f(x, \omega), x) \mathbb{P}(d \omega) \mu(d x)<\infty
$$

and $P\left(\mathcal{M}_{1}^{1}(X)\right) \subset \mathcal{M}_{1}^{1}(X)$.

Proof. Fix $\varphi \in \operatorname{Lip}_{1}^{b}(X)$. Then (see Remark 5.2)

$$
\begin{aligned}
\left|\int \varphi d P \mu-\int \varphi d \mu\right| & =\left|\int\left(P^{*} \varphi-\varphi\right) d \mu\right| \\
& \leq \int_{X}\left|\int_{\Omega} \varphi(f(x, \omega)) \mathbb{P}(d \omega)-\varphi(x)\right| \mu(d x) \\
& \leq \int_{X} \int_{\Omega} \rho(f(x, \omega), x) \mathbb{P}(d \omega) \mu(d x)<\infty .
\end{aligned}
$$

This shows (5.4). Moreover, by (5.2) we have $\left|P^{*} \varphi(x)-P^{*} \varphi(y)\right| \leq \lambda \rho(x, y)$ for $x, y \in X$, and the use of Corollary 4.5 ends the proof.

Remark 5.4. Assume that the operator $P$ is given by (2.1), condition (5.2) is fulfilled for some $\lambda \in(0,1)$ and $P\left(\mathcal{M}_{1}^{1}(X)\right) \subset \mathcal{M}_{1}^{1}(X)$ or equivalently $d_{H}\left(P \delta_{x_{0}}, \delta_{x_{0}}\right)<\infty$ for some (thus all) $x_{0} \in X$. Then for every $x_{0} \in X$ we have

$$
d_{H}\left(P \delta_{x_{0}}, \delta_{x_{0}}\right)=\int_{\Omega} \rho\left(f\left(x_{0}, \omega\right), x_{0}\right) \mathbb{P}(d \omega) ;
$$

in particular (5.3) holds.

Proof. Fix $x_{0} \in X$ and define $V \in \operatorname{Lip}_{1}(X)$ by (3.2). Clearly $P \delta_{x_{0}} \in \mathcal{M}_{1}^{1}(X)$ and according to Lemma 3.1.(i) we obtain

$$
\begin{aligned}
\infty & >d_{H}\left(P \delta_{x_{0}}, \delta_{x_{0}}\right)=D_{H}\left(P \delta_{x_{0}}, \delta_{x_{0}}\right) \\
& =\sup \left\{\int \varphi d P \delta_{x_{0}}-\int \varphi d \delta_{x_{0}}: \varphi \in \operatorname{Lip}_{1}(X)\right\} \\
& \geq\left|\int V d P \delta_{x_{0}}-\int V d \delta_{x_{0}}\right|=\int V d P \delta_{x_{0}} \\
& =\int P^{*} V(x) \delta_{x_{0}}(d x)=\int_{\Omega} \rho\left(f\left(x_{0}, \omega\right), x_{0}\right) \mathbb{P}(d \omega) ;
\end{aligned}
$$

in particular (5.3) is fulfilled. Having (5.3) we can apply (5.4) to get (5.5). 
Theorem 5.5. Assume that (5.2) and (5.3) hold for some $\lambda \in(0,1)$ and $x_{0} \in X$. Then the operator $P$ given by (2.1) is asymptotically stable and its invariant measure $\mu^{*}$ belongs to $\mathcal{M}_{1}^{1}(X)$. Moreover,

$$
d_{H}\left(P^{n} \mu, \mu^{*}\right) \leq \frac{\lambda^{n}}{1-\lambda} \int_{X} \int_{\Omega} \rho(f(x, \omega), x) \mathbb{P}(d \omega) \mu(d x)
$$

for any $n \in \mathbb{N}$ and $\mu \in \mathcal{M}_{1}^{1}(X)$.

Proof. Clearly (4.1) holds, by (5.2). Due to Lemma 5.3 we get (4.2) with any $\mu_{0} \in \mathcal{M}_{1}^{1}(X)$. To finish the proof it is enough to apply Theorem 4.1 and Lemma 5.3 again.

On account of (5.1), we conclude that the asymptotic stability of $P$ given by (2.1) implies that for every $x \in X$ the sequence $\left(f^{n}(x, \cdot)\right)$ converges in distribution even when $P$ does not have the Feller property (cf. Remark 5.1). Due to this fact and Theorem 5.5 we have the following corollary.

Corollary 5.6. Assume that (5.2) and (5.3) hold for some $\lambda \in(0,1)$ and $x_{0} \in X$. Then for every $x \in X$ the sequence of iterates $\left(f^{n}(x, \cdot)\right)$ converges in distribution and the limit $\mu^{*}$ does not depend on $x$. Moreover, $\mu^{*} \in \mathcal{M}_{1}^{1}(X)$ and

$$
\begin{aligned}
d_{H}\left(\pi_{n}(x, \cdot), \mu^{*}\right) & \leq \frac{\lambda^{n}}{1-\lambda} \int_{\Omega} \rho(f(x, \omega), x) \mathbb{P}(d \omega) \\
& \leq \frac{\lambda^{n}}{1-\lambda}\left((\lambda+1) \rho\left(x, x_{0}\right)+\int_{\Omega} \rho\left(f\left(x_{0}, \omega\right), x_{0}\right) \mathbb{P}(d \omega)\right)
\end{aligned}
$$

for any $n \in \mathbb{N}$ and $x \in X$.

\section{Acknowledgements}

This work was partially supported by the Faculty of Applied Mathematics AGH UST statutory tasks within subsidy of Ministry of Science and Higher Education.

Open Access. This article is distributed under the terms of the Creative Commons Attribution 4.0 International License (http://creativecommons.org/licenses/by/4.0/), which permits unrestricted use, distribution, and reproduction in any medium, provided you give appropriate credit to the original author(s) and the source, provide a link to the Creative Commons license, and indicate if changes were made.

\section{References}

[1] Baron, K.: On the convergence in law of iterates of random-valued functions. Aust. J. Math. Anal. Appl. 6, 1-9 (2009)

[2] Baron, K.: Weak law of large numbers for iterates of random-valued functions. Aequat. Math. (2018). https://doi.org/10.1007/s00010-018-0585-0 
[3] Baron, K., Jarczyk, W.: Random-valued functions and iterative functional equations. Aequat. Math. 67, 140-153 (2004)

[4] Baron, K., Kuczma, M.: Iteration of random-valued functions on the unit interval. Colloq. Math. 37, 263-269 (1977)

[5] Diamond, P.: A stochastic functional equation. Aequat. Math. 15, 225-233 (1977)

[6] Dudley, R.M.: Real Analysis and Probability. Cambridge Studies in Advanced Mathematics, vol. 74. Cambridge Univerity Press, Cambridge (2002)

[7] Fortet, R., Mourier, E.: Convergence de la répartition empirique vers la répartition théorique. Ann. Sci. Ecole Norm. Sup. 70, 267-285 (1953)

[8] Hutchinson, J.: Fractals and self-similarity. Indiana Univ. Math. J. 30, 713-747 (1981)

[9] Kapica, R.: Random iteration and Markov operators. J. Differ. Equ. Appl. 22, 295-305 (2016)

[10] Lasota, A.: From fractals to stochastic differential equations. Lect. Notes Phys. 457, 235-255 (1995)

[11] Villani, C.: Optimal transport. Old and new. In: Grundlehren der Mathematischen Wissenschaften [Fundamental Principles of Mathematical Sciences], vol. 338, Springer, Berlin (2009)

Rafał Kapica

Faculty of Applied Mathematics

AGH University of Science and Technology

30-059 Kraków

Poland

e-mail: rafal.kapica@agh.edu.pl

Received: March 29, 2018

Revised: October 22, 2018 